\title{
Hemobilia as a rare complication after endoscopic ultrasound-guided fine-needle aspiration for hilar cholangiocarcinoma
}

Endoscopic ultrasound-guided fine-needle aspiration (EUS-FNA) is a useful and safe procedure for obtaining tissue samples for the diagnosis of lesions adjacent to the gastrointestinal tract $[1,2]$. We describe a first case of hemobilia presenting as obstructive jaundice after EUS-FNA for hilar cholangiocarcinoma.

A 67-year-old man presented at our institution with malaise. Abdominal magnetic resonance imaging (MRI) and EUS (EG530 UT2; Fujifilm, Tokyo, Japan) showed a hilar bile duct tumor ( Fig. 1); however, the patient's serum bilirubin level was normal.

For obtaining tissue samples, we performed EUS-FNA on the hilar bile duct tumor from the duodenal bulb by using a 22gauge needle (Echotip-Ultra; WilsonCook, Winston-Salem, North Carolina, USA). The pathological specimen revealed adenocarcinoma, and the patient was diagnosed with hilar cholangiocarcinoma. After 4 days, he complained of abdominal pain and icterus. A laboratory test revealed a serum bilirubin level of $6.6 \mathrm{mg} / \mathrm{dL}$. Abdominal computed tomography (CT) showed that high-density fluid was retained in the gallbladder and common bile duct ( $\bullet$ Fig. 2 ).

We suspected obstructive jaundice caused by hemobilia. A duodenoscope showed that blood was flowing out from the papilla ( $\bullet$ Fig. 3).

We placed a 5-Fr endoscopic nasobiliary drainage (ENBD) tube for biliary drainage. Hemobilia subsided following conservative therapy, and the ENBD tube was removed after 8 days.

Hemobilia is a rare complication after percutaneous liver biopsy [3]. The accidental laceration of an artery or a portal vein is considered to be the likely cause of hemobilia. Although EUS-FNA of bile duct tumors has been reported frequently [4], there were no reports of hemobilia as a complication of EUS-FNA. In our patient,

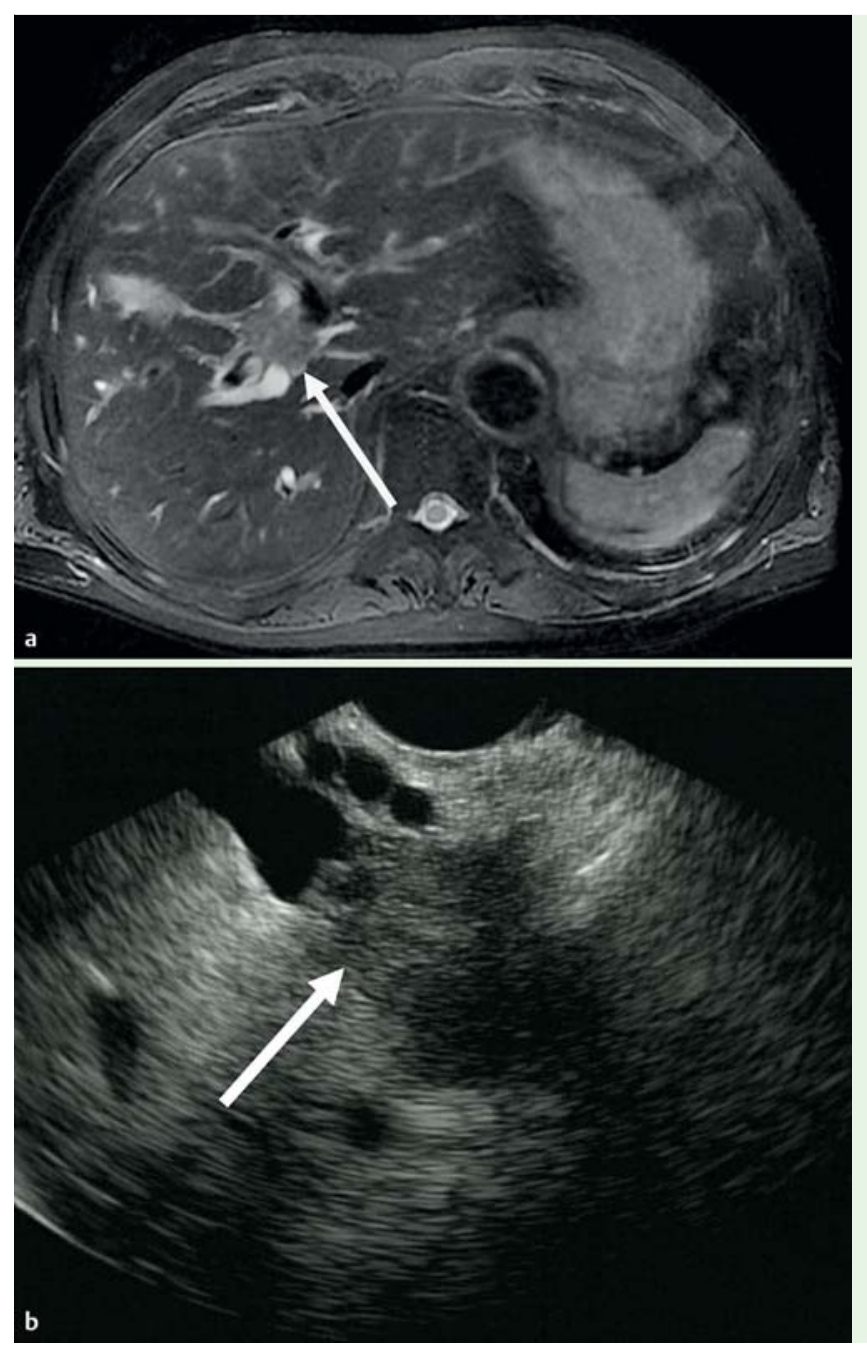

Fig. 1 a Magnetic resonance image showing a low-intensity mass at the hilar region (arrow). b Endoscopic ultrasound (EUS) image showing hypoechoic tumor at the hilar bile duct (arrow).

hemobilia occurred 4 days after EUS-FNA, which was within the previously reported range in which hemobilia developed after liver biopsy [3]. Endoscopic retrograde cholangiopancreatography (ERCP) can successfully visualize blood coming out from the papilla. Although, in our case, hemobilia resolved with ENBD tube insertion, transarterial embolization or surgery may be sometimes necessary for its management [5].
Endoscopy_UCTN_Code_CPL_1AL_2AF

Competing interests: None

K. Kawakubo, H. Isayama,

N. Takahara, N. Yamamoto,

H. Kogure, T. Sasaki, K. Hirano,

N. Sasahira, M. Tada, K. Koike

Department of Gastroenterology,

Graduate School of Medicine,

The University of Tokyo, Japan 


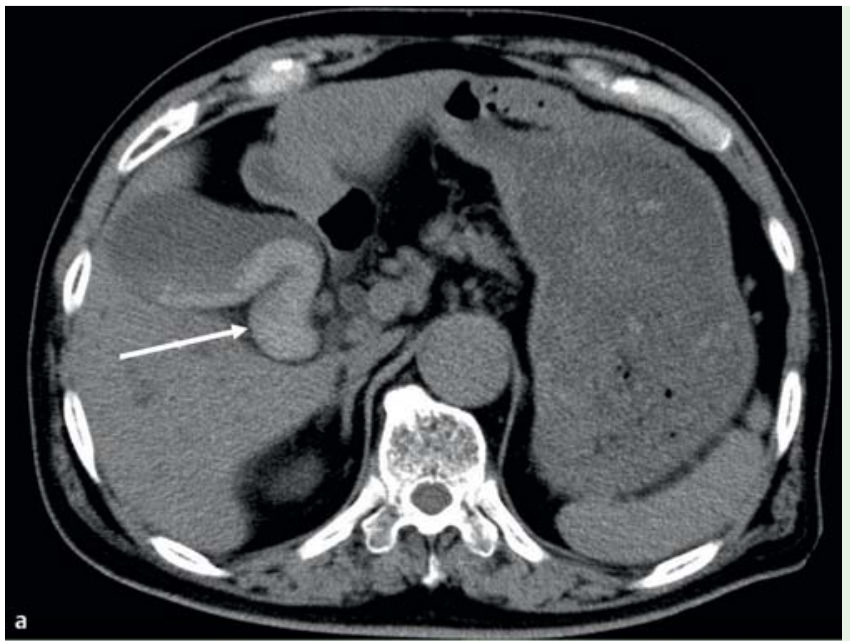

Fig. 2 Abdominal computed tomography (CT) showing highdensity fluid in: a the gallbladder (arrow) and b the lower bile duct (arrow).

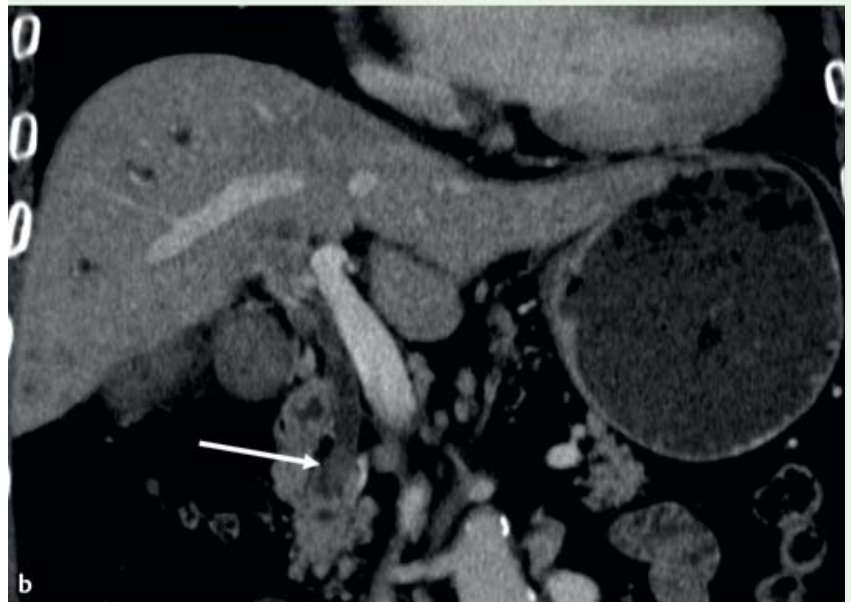

\section{References}

1 Wiersema MJ, Vilmann P, Giovannini M et al. Endosonography-guided fine-needle aspiration biopsy: diagnostic accuracy and complication assessment. Gastroenterology 1997; 112: 1087-1095

2 Wang KX, Ben QW, Jin ZD et al. Assessment of morbidity and mortality associated with EUS-guided FNA: a systematic review. GastrointestEndosc 2011; 73: $283-290$

3 Piccinino F, Sagnelli E, Pasquale G et al. Complications following percutaneous liver biopsy. A multicentre retrospective study on 68,276 biopsies. J Hepatol 1986; 2: $165-$ 173

4 Fritscher-Ravens A, Broering DC, Knoefel WT et al. EUS-guided fine-needle aspiration of suspected hilarcholangiocarcinoma in potentially operable patients with negative brush cytology. Am J Gastroenterol 2004; 99: $45-51$

5 Green MH, Duell RM, Johnson CD et al. Haemobilia. Br J Surg 2001; 88: 773-786
Bibliography

DOI $10.1055 / \mathrm{s}-0030-1256783$

Endoscopy 2011; 43: E334-E335

(c) Georg Thieme Verlag KG Stuttgart - New York . ISSN 0013-726X

\section{Corresponding author}

\section{K. Kawakubo, MD}

Department of Gastroenterology

Graduate School of Medicine

The University of Tokyo

7-3-1 Hongo Bunkyo-ku

Tokyo

113-8655

Japan

Fax: +81-3-38140021

kkawakubo-gi@umin.ac.jp

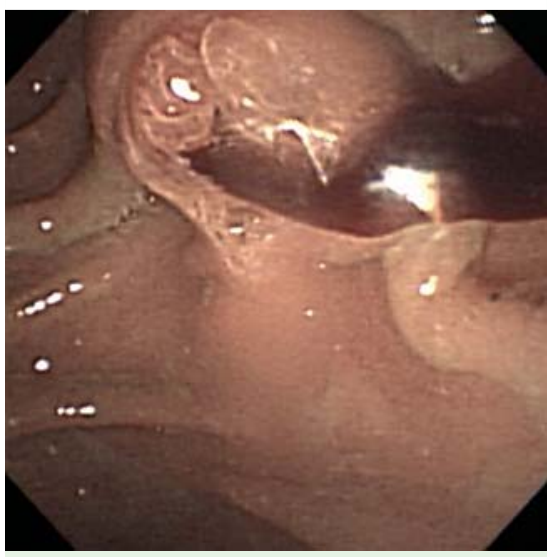

Fig. 3 Endoscopic image showing blood coming out from the papilla. 